\title{
A general formula for computing maximum proportion correct scores in various psychophysical paradigms with arbitrary probability distributions of stimulus observations
}

\author{
Huanping Dai • Christophe Micheyl
}

Published online: 28 February 2015

(C) The Psychonomic Society, Inc. 2015

\begin{abstract}
Proportion correct $(P c)$ is a fundamental measure of task performance in psychophysics. The maximum $P c$ score that can be achieved by an optimal (maximum-likelihood) observer in a given task is of both theoretical and practical importance, because it sets an upper limit on human performance. Within the framework of signal detection theory, analytical solutions for computing the maximum $P c$ score have been established for several common experimental paradigms under the assumption of Gaussian additive internal noise. However, as the scope of applications of psychophysical signal detection theory expands, the need is growing for psychophysicists to compute maximum $P c$ scores for situations involving non-Gaussian (internal or stimulus-induced) noise. In this article, we provide a general formula for computing the maximum $P c$ in various psychophysical experimental paradigms for arbitrary probability distributions of sensory activity. Moreover, easy-to-use MATLAB code implementing the formula is provided. Practical applications of the formula are illustrated, and its accuracy is evaluated, for two paradigms and two types of probability distributions (uniform and Gaussian). The results demonstrate that $P c$ scores computed using the formula remain accurate even for continuous probability distributions, as long as the conversion from continuous probability density functions to discrete probability mass functions is supported by a sufficiently high sampling resolution. We hope that the exposition in this article, and the freely available MATLAB code, facilitates calculations of maximum performance for a wider range of experimental situations, as
\end{abstract}

H. Dai $(\square)$

Department of Speech, Language, and Hearing Sciences,

College of Science, University of Arizona, 1131 E. 2nd Street,

Tucson, AZ 85721, USA

e-mail: hdai@email.arizona.edu

C. Micheyl

Starkey Hearing Research Center, Berkeley, CA, USA well as explorations of the impact of different assumptions concerning internal-noise distributions on maximum performance in psychophysical experiments.

Keywords Proportion correct · Psychophysics · Optimal performance $\cdot$ Signal detection theory $\cdot$ Internal noise

The most commonly used measure of performance for participants in psychophysical experiments is the proportion of correct $(P c)$ responses. Within the framework of signal detection theory (SDT; Green \& Swets, 1966), researchers often transform $P c$ scores measured in a psychophysical experiment into the unbiased index of sensitivity, $d^{\prime}$, after taking into account any possible response bias of the participants. Mathematical formulas and tables for converting $P c$ into $d^{\prime}$ have been provided in earlier publications for the most commonly used psychophysical paradigms, including yes-no, same-different, multiple-alternative forced choice (mAFC), dual-pair comparison (e.g., 4IAX), and oddity (Green \& Swets, 1966; Macmillan \& Creelman, 2005). However, these formulas and tables are almost invariably based on the assumption of Gaussian-distributed, constant-variance, additive, internal (observer) or external (stimulus) noise.

Although the equal-variance Gaussian noise assumption is mathematically convenient and is sometimes justified by the central-limit theorem, there are many situations in which this assumption is inadequate. These include, for instance, experiments in which the characteristics of either the stimuli or the sensory process lead to sensory observations that are nonGaussian, or have unequal variances. A well-known example is the measurement of visual detection thresholds, which have been shown to depend on the number of photons - a Poissondistributed random variable - that hit the retina (Hecht, Shlaer, \& Pirenne, 1942). A second example comes from 
experiments concerning the discrimination of differences in the "width," or variance, of stimuli (see, e.g., Creelman, 1962, Geisler, Albrecht, Salvi, \& Saunders, 1991; Lutfi \& Doherty, 1994; and Lutfi, Doherty, \& Oh, 1996, for auditory stimuli; and Geisler et al., 1991; Graham, Kramer, \& Yager, 1987; and Rieke, Warland, de Ruyter van Steveninck, \& Bialek, 1999, for visual stimuli). Even for tasks in which the equal-variance Gaussian assumption is routinely made, detailed examinations of empirical data, including receiveroperating characteristics (ROCs), have indicated that models involving unequal variances or non-Gaussian distributions provide a more satisfactory account of the data (e.g., Graham et al., 1987; Green \& Swets, 1966; Macmillan \& Creelman, 2005; May \& Solomon, 2013; Micheyl, Kaernbach, \& Demany, 2008; Nachmias \& Kocher, 1970; Neri, 2013). Yet another example of situations in which the Gaussian assumption is inadequate relates to experiments in which a stimulus feature, which could provide an unwanted cue for task performance, is randomly perturbed (or "roved") across observation intervals within a trial, or across trials, in order to limit its use by participants. Examples of this abound in both the auditory-perception literature (e.g., Berg \& Green, 1990; Berliner \& Durlach, 1973; Dai, 1994, 2008; Dai \& Green, 1993; Dai, Nguyen, \& Green, 1995, 1996; Hall \& Fernandes, 1983; Henning, 1966; Jesteadt \& Bilger, 1974; Kidd, Mason, Brantley, \& Owen, 1989; Kidd, Mason, Uchanski, Brantley, \& Shah, 1991; Micheyl, Divis, Wrobleski, \& Oxenham, 2010; Moore \& Glasberg, 1989; Oxenham \& Buus, 2000; Semal \& Demany, 2006; Spiegel, Picardi, \& Green, 1981; Strickland \& Dhar, 2000) and the visual-perception literature (e.g., Danilova \& Mollon, 2010; Dannemiller \& Stephens, 1998; Morgan, 2005; Nachmias, 1999, 2002; Regan \& Hamstra, 1991, 1992; Webster, De Valois, \& Switkes, 1990). In such "roving" experiments, the experimenter must determine the size of the roving range necessary to ensure that the highest $P c$ that participants can achieve using the unwanted cue is below a predefined target (e.g., $60 \%$ correct). In order to do this, the experimenter needs formulas for computing $P c$ that can take into account the influence of the "unwanted" cue on the distance between the means of the two distributions of sensory observations that the observer must compare in order to respond correctly in the task (see Dai \& Kidd, 2009; Dai \& Micheyl, 2010, 2012; Green, 1988). Since, in the vast majority of studies that have made use of stimulus randomization, the random stimulus perturbations are uniformly distributed, formulas for computing $P c$ based on the assumption of Gaussian-distributed observations cannot be applied (e.g., Dai \& Micheyl, 2012; Green, 1988).
As the examples above illustrate, as the scope of applications of psychophysical SDT expands, the need is growing for experimenters to relate measures of participants' performance, such as $P c$, to the statistical properties of the stimulus or sensory observations in situations that involve non-Gaussian distributions. Toward this aim, researchers currently have two main approaches at their disposal. The first involves trying to find an analytical solution for the specific probability distribution (or family of probability distributions) at hand. This can sometimes be achieved, especially if an analytical formula for the probability distribution of interest already exists, or if that probability distribution can be approximated by a Gaussian distribution - see, for instance, the derivations of $P c$ for the energy detector using Chi-square distributions, and for the envelope detector using Rayleigh distributions (Green \& Swets, 1966). In general, analytical solutions are desirable because, when such a solution is available, it can yield unique insights into the influence of model parameters on the results, and can be used to obtain accurate, rather than approximate, results. In practice, however, closed-form, analytical solutions are often intractable or, at least, arduous.

A second approach relies on simulations. In this approach, the researcher typically simulates the sensory and decision processes of an optimal (usually, maximum-likelihood) observer in a virtual experiment that has characteristics similar to those of an actual psychophysical experiment over a large number of trials. This approach has gained popularity in the past decades, owing to the widespread availability of fast processors, large memory sizes, and computational software. However, it has some limitations. Firstly, the approach requires that random samples be drawn from the target distribution. If the distribution does not pertain to a family for which a random-number generator already exists in the software packages at hand, the researcher must use more elaborate computational techniques for drawing samples from nonstandard distributions, which can be more difficult to implement owing to their complexity. Secondly, assuming that an adequate random-number generator is available, the researcher must formulate explicitly the observer's decision rule in order to simulate the decision process. Usually, the researcher is interested primarily in the best possible decision strategy (the optimal decision rule), because this provides an upper bound on performance against which human performance can be compared. For equal-variance Gaussian observations, the maximum-likelihood (ML) decision rule often turns out to be fairly straightforward, typically involving a comparison between a random variable and a scalar, or between two or more random variables with, sometimes, an absolute-value operator. However, less common distributions can lead to more complicated decision rules, especially if the 
experimental paradigm involves more than two observation intervals, or multiple response alternatives.

The purpose of this article is to describe a third approach to computing maximum $P c$ scores, which is intermediate between the above-mentioned "analytical-solution" and "Monte Carlo simulation" approaches. As we show in the next, Analysis and discussion section, the approach is derived from the definition of the ML decision strategy (the optimal decision rule). Relative to the first two approaches, the new approach has some unique merits. Since it is not based on simulation, it avoids some of the limitations of the Monte Carlo approach mentioned above. Likewise, although the new approach does not measure up to the analytical-solution approach when such a solution is available - because it does not readily yield the same quality of insight that a true analytical solution can yield - we believe that it can partly make up for this shortcoming by providing researchers with a practical, accurate, and easily accessible tool for dealing with situations that fall outside the standard Gaussian framework.

In this article, we provide general equations into which researchers can simply "plug" whatever particular probability distributions they wish to use to obtain computations of optimal performance-the maximum $P c$ score. These equations can be used to compute the maximum $P c$ scores for various experimental paradigms. In each paradigm, all response alternatives or events are expressed in terms of two basic stimulus events, denoted as $\mathrm{A}$ and $\mathrm{B}$, which may be regarded as the building blocks for constructing joint stimulus events, which occur in other paradigms. These basic stimulus events can correspond, for example, to the presentation of "noise-alone" (B) and "signal-plus-noise" (A) stimuli in a yes-no task. The probability distribution functions of the two events are referred to as the basis functions, denoted as $f_{x}(\boldsymbol{x} \mid A)$ and $f_{x}(\boldsymbol{x} \mid B)$, in which the vector $\boldsymbol{x}$ represents the stimulus observation variable. For other paradigms, the joint probability distributions of the joint stimulus events are multidimensional and are constructed using these two basis functions. In the Appendix, we provide the MATLAB code of the general formula. To the code the user can input the two basis probability distributions $f_{x}(\boldsymbol{x} \mid A)$ and $f_{x}(\boldsymbol{x} \mid B)$ in the form of two vector functions of equal length, along with a selection of the psychophysical paradigm. If the selection is the yes-no paradigm, then the code will compute the maximum $P c$ score directly from $f_{x}(\boldsymbol{x} \mid A)$ and $f_{x}(\boldsymbol{x} \mid B)$. If the selection is a paradigm comprising $m$ joint stimulus events, the code will first build all $m$ multidimensional joint probability distributions from $f_{x}(\boldsymbol{x} \mid A)$ and $f_{x}(\boldsymbol{x} \mid B)$, and then compute the maximum $P c$ score from the $m$ joint probability distributions.

\section{Analysis and discussion}

In this section, we describe the general features of the mathematical formula that can be used to compute the maximum proportions correct, $P c$, for various commonly used experimental paradigms. These experimental paradigms are defined in terms of the basis probability distributions $f_{x}(\boldsymbol{x} \mid A)$ and $f_{x}(\boldsymbol{x} \mid B)$ of the two basic stimulus events, A and B. Features of the formula that are specific to each paradigm are described in a separate subsection below. The formula is consistent with fundamental principles of statistical decision theory and, more specifically, with basic model of psychophysical SDT (Green \& Swets, 1966). Using this model, optimal (ML) decision strategies for the various paradigms have been analyzed in earlier publications, almost invariably assuming Gaussiandistributed sensory observations (e.g., Dai \& Kidd, 2009; Dai \& Micheyl, 2010, 2012; Dai, Versfeld, \& Green, 1996; Green \& Dai, 1991; Green \& Swets, 1966; Irwin \& Hautus, 1997; Irwin, Hautus, \& Butcher, 1999; Macmillan \& Creelman, 2005; Micheyl \& Dai, 2008, 2009; Micheyl et al., 2008; Micheyl \& Messing, 2006; Noreen, 1981; Rousseau \& Ennis, 2001, 2002; Versfeld, Dai, \& Green, 1996). Readers who are interested in details are referred to these earlier publications. Here, we only list the general assumptions of the SDT model, provide a brief description of each paradigm, and give the equation that can be used for determining the maximum $P c$ for the paradigm under consideration with arbitrary probability distributions.

According to the SDT model (Green \& Swets, 1966), each stimulus presentation yields an "observation," which we denote as $x$, and on this basis the decision maker decides between or among response alternatives. We assume that the probability distributions of the observations are known to the researcher, so that the researcher can plug these distributions into the formulas to compute the maximum $P c$ values. Depending on which psychophysical paradigm is used, $n$ stimuli $(n=1,2,3$, etc.) may be presented on each trial. The corresponding $x$ values on a trial are denoted as $x_{1}, \ldots, x_{n}$. For notational convenience, we define the vector of observations as $\boldsymbol{x}=\left[x_{1}, \ldots, x_{n}\right]$.

We are interested in determining the maximum $P c$ that can be achieved by an observer who responds on each trial solely on the basis of the vector of observations, $\boldsymbol{x}$. It is known that the proportion correct is maximized by using a likelihoodratio decision strategy (Green \& Swets, 1966), which involves choosing the response alternative corresponding to the most likely stimulus event, given the single stimulus (when $n=1$ ) or stimulus sequence (when $n \geq 2$ ) that has been presented on the current trial. For example, in the same-different paradigm there are four possible stimulus sequences- $\mathrm{AA}, \mathrm{BB}, \mathrm{AB}$, and 
BA; the former two sequences are associated with a "same" response, and the latter two with a "different" response. On a trial, the optimal decision is to respond "same" if and only if the joint probability of the sequences $\mathrm{AA}$ or $\mathrm{BB}$ is greater than that of the sequences $\mathrm{AB}$ or $\mathrm{BA}$. In the more general case, we have $m$ response alternatives, labeled $H_{1}, \ldots, H_{m}$. Let $E_{i}$ denote the stimulus event corresponding to the occurrence of all stimulus sequences associated with the $i$ th response alternative $\left(H_{\mathrm{i}}\right)$, and $P\left(E_{i}\right)$ the a priori probability of $E_{i}$. For a specific presentation of the observation vector $\boldsymbol{x}$, let $P\left(\boldsymbol{x} \mid E_{i}\right)$ denote the conditional probability of $\boldsymbol{x}$ given event $E_{i}$, and $P(\boldsymbol{x})$ the probability of $\boldsymbol{x}$. By Bayes's rule, the conditional (a posteriori) probability of the stimulus event $E_{i}$, given the presentation of $\boldsymbol{x}$, is $P\left(E_{i} \mid x\right)=\left[P\left(x \mid E_{i}\right) P\left(E_{i}\right)\right] / \sum_{i=1}^{m} P\left(x \mid E_{i}\right) P\left(E_{i}\right)$. According to the ML decision strategy, the observer should choose response $H_{i}$ if $P\left(E_{i} \mid x\right)=\max \left\{P\left(E_{i} \mid x\right), \ldots, P\left(E_{m} \mid x\right)\right\}$, or $P\left(E_{i} \mid x\right)=\max \left\{P\left(x \mid E_{1}\right) P\left(E_{1}\right), \ldots, P\left(x \mid E_{m}\right) P\left(E_{m}\right)\right\}$
$/ \sum_{i=1}^{m} P\left(x \mid E_{i}\right) P\left(E_{i}\right)$. The probability that this response is correct can be expressed as $P c(x)=P\left(E_{i} \mid x\right) / \sum_{j=1}^{m} P\left(E_{j} \mid x\right)$. Since $\sum_{j=1}^{m} P\left(E_{j} \mid x\right)=1, \quad$ w e have $P c(x)=P\left(E_{i} \mid x\right)=\max$ $\left\{P\left(x \mid E_{1}\right) P\left(E_{1}\right), \ldots, P\left(x \mid E_{m}\right) P\left(E_{m}\right)\right\} / \sum_{i=1}^{m} P\left(x \mid E_{i}\right) P\left(E_{i}\right)$. The expected value of $P c(x)$, which is the maximum proportion correct score that we are deriving, can be obtained by integrating the expression above over all possible values of the observation vector $\boldsymbol{x}$. Let $f_{x}\left(x \mid E_{i}\right)$ denote the conditional probability density function (PDF) of $\boldsymbol{x}$ given event $E_{i}$, and $f_{x}(x)$ the PDF of $\boldsymbol{x}$. [Note that both $f_{x}\left(x \mid E_{i}\right)$ and $f_{x}(x)$ are joint PDFs except when $n=1$, as in a yes-no task.] By substituting these PDFs for the probabilities in the expression of $P c(x)$ and integrating $P c(x)$, we obtain the general formula for computing the maximum proportion correct score as

$P c_{m}=\int_{x} P c(x) f_{x}(x) d x=\int_{x_{n}} \ldots \int_{x_{2}} \int_{x_{1}} \frac{\max \left\{f_{x}\left(x \mid E_{1}\right) P\left(E_{1}\right), \ldots, f_{x}\left(x \mid E_{m}\right) P\left(E_{m}\right)\right\}}{\sum_{i=1}^{m} f_{x}\left(x \mid E_{i}\right) P\left(E_{i}\right)} f_{x}(x) d x_{1} d x_{2} \ldots d x_{n}$

Although Eq. 1 is derived under the assumption that the observation vector is a continuous variable, it can be readily modified and extended to discrete variables; we return to this point below. Equation 1 can be further simplified using two assumptions commonly made in psychophysical applications. First, assuming that the different stimulus events are mutually exclusive, we have $f_{x}(x)=\sum_{i=1}^{m} f_{x}\left(x \mid E_{i}\right) P\left(E_{i}\right)$. Equation 1
then simplifies to

$P c_{m}=\int_{x_{n}} \ldots \int_{x_{2}} \int_{x_{1}} \max \left\{f_{x}\left(x \mid E_{1}\right) P\left(E_{1}\right), \ldots, f_{x}\left(x \mid E_{m}\right) P\left(E_{m}\right)\right\} d x_{1} d x_{2} \ldots d x_{n}$.

Second, assuming that the events, $E_{i}, \ldots, E_{m}$ all have the same probability of occurrence a priori, we have $P\left(E_{1}\right)=P\left(E_{2}\right)=\ldots=P\left(E_{m}\right)=1 / m$. In such cases, Eq. 2 simplifies to

$P c_{m}=\frac{1}{m} \int_{x_{n}} \ldots \int_{x_{2}} \int_{x_{1}} \max \left\{f_{x}\left(x \mid E_{1}\right), \ldots, f_{x}\left(x \mid E_{m}\right)\right\} d x_{1} d x_{2} \ldots d x_{n}$.

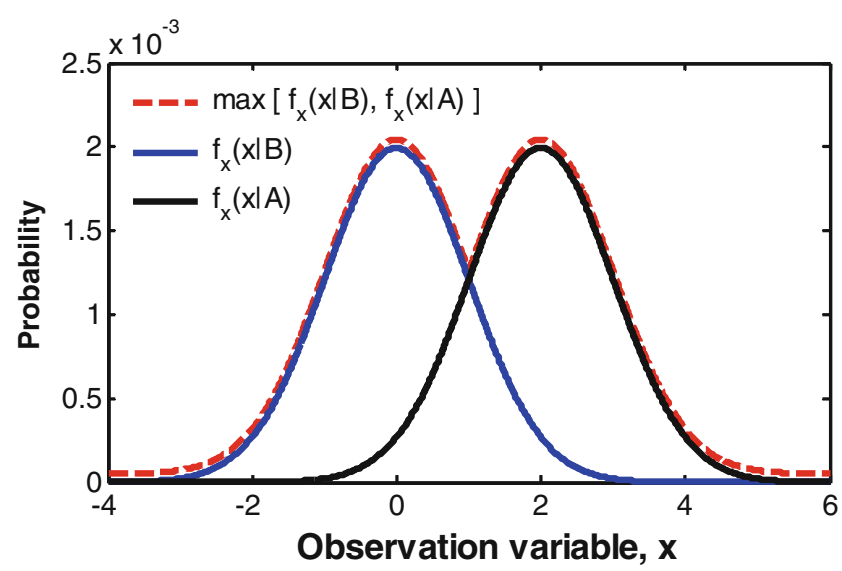

Fig. 1 Visual example illustrating the terms of Eq. 3 in general, and of Eq. 4, $P c_{y e s-n o}=\frac{1}{2} \int_{x} \max \left[f_{x}(x \mid A), f_{x}(x \mid B)\right] d x$, in particular, used for computing the maximum proportion correct $(P c)$ score for a yes-no paradigm. The solid gray (blue in the online version) curve, or $f_{x}(\boldsymbol{x} \mid B)$, represents the probability density function (PDF) of the $<\mathrm{n}>$ (noise-alone) event $(\mu=0)$; the solid black curve, or $f_{x}(\boldsymbol{x} \mid A)$, represents the PDF of the $<\mathrm{S}>$ (signal + noise) event; and the dashed curve (which has been elevated slightly for visual clarity), or $\max \left[f_{x}(\boldsymbol{x} \mid A), f_{x}(\boldsymbol{x} \mid B)\right]$, represents the maximum of the two density functions $f_{x}(\boldsymbol{x} \mid A)$ and $f_{x}(\boldsymbol{x} \mid B)$. The maximum $P c$ is computed as half of the size of the area under the dashed curve 
The general formula in Eq. 3 relates the maximum proportion correct to the PDFs of the stimulus or observation variables, conditioned on different trial types. The exact form of the conditional PDF, $f_{x}\left(\boldsymbol{x} \mid E_{i}\right)$, depends on the forms of the two basis functions and on the psychophysical paradigm used. Therefore, the specific formula for computing the maximum $P c$ derived from Eq. 3 takes different specific forms, depending on the paradigm. In Fig. 1, we provide an example to visually illustrate the probabilities involved in computing the maximum $P c$ score using Eq. 3.

Next, in Section A, we present the detailed expressions of Eq. 3 for seven representative paradigms, including the yesno, two- and three-interval forced choice (2IFC and 3IFC), same-different, 3I- and 4I-oddity, and dual-pair comparison paradigms. It is important to note that the formula can be readily modified to accommodate discrete distributions by simply replacing the probability density function of the continuous distributions with the probability mass functions (PMFs) of the discrete distributions and changing the integration to summation - both modifications are straightforward. This point is important, because in many potential applications of the general formula (Eqs. 1, 2, or 3) in psychophysics, the probability distributions of the sensory observations will be discrete rather than continuous. In fact, in our implementation of the general formula (Eq. 3) in MATLAB code, which is provided in the Appendix, the input probability distributions must be discrete functions (i.e., PMFs). When the MATLAB code of the general formula is used to compute the maximum $P c$ for cases in which the probability distributions are continuous functions, the researcher will need to convert the continuous PDFs into discrete PMFs. As we illustrate in Section B below, the computed $P c$ scores for continuous cases are highly accurate as long as the converted discrete PMFs have sufficiently large numbers of bins (i.e., the sampling resolution is sufficiently high) to closely approximate the original continuous PDFs. It is also worth noting that the formula remains true regardless of whether the elements $\left(x_{1}, \ldots, x_{n}\right)$ in the observation vector $\boldsymbol{x}$ are correlated with each other over trials - that is, regardless of the properties of the covariance matrix of $\boldsymbol{x}$. To illustrate how to apply the formulas associated with the seven paradigms to specific probability distributions, for each paradigm we specified the formula to the familiar Gaussian distributions, as a concrete example.

In Section B, we evaluate the accuracy and applicability of the present procedure for computing the maximum $P c$ score using the general formula in Eq. 3. In particular, we applied the formula to the 2IFC paradigm in the context of continuous Gaussian distributions, and to the 3IFC paradigm in the context of discrete uniform distributions. We chose these two combinations of paradigms and probability distributions because for both cases, the analytical solutions for computing $P c$

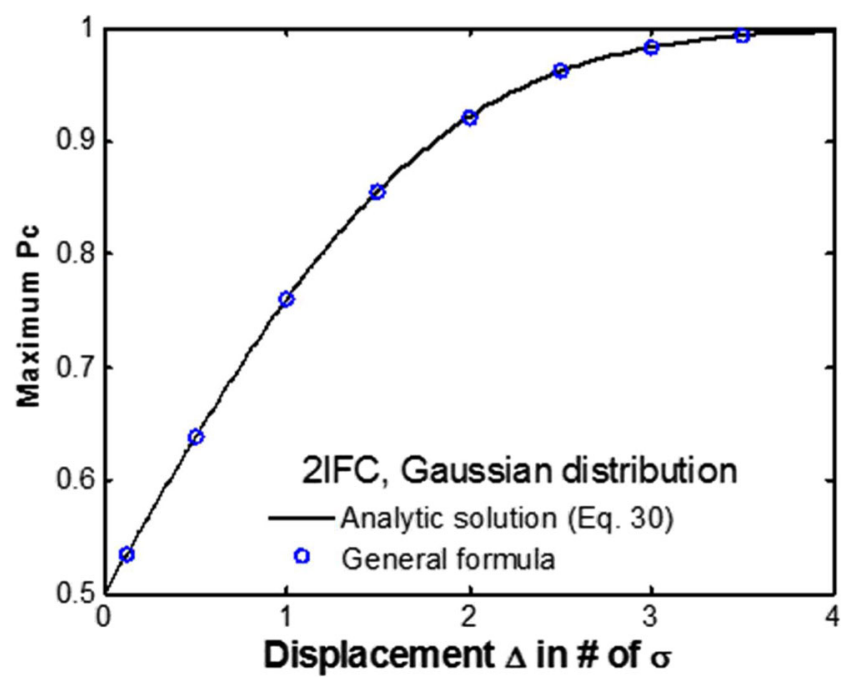

Fig. 2 Maximum proportion correct $(P c)$ scores as a function of displacement $(\Delta)$ for the 2IFC paradigm under a continuous Gaussian distribution. The $\Delta$ value between the probability distributions of the noise-alone event (B) and the signal + noise event (A) is expressed in units of the standard deviation $(\sigma)$ of the Gaussian distribution. The circles represent the $P c$ scores computed using the general formula. For this computation, the probability mass functions converted from the probability density functions are represented by a sampling resolution of 200 bins per $\sigma$. The solid curve represents the $P c$ scores computed using the standard analytical solution from signal detection theory under the assumption of Gaussian distributions with equal variances. Under this assumption, $\Delta$ is identical to the detectability index, $d^{\prime}$

are available in the SDT literature (see Green \& Swets, 1966, for the case of 2IFC under Gaussian distributions, and Dai \& Kidd, 2009, for the case of 3IFC under uniform distributions.) Furthermore, we included the uniform distribution in this evaluation so that we would have examined at least one nonGaussian example. In each case of our evaluation, we compared the $P c$ values computed using either the analytic solutions of SDT or the present procedure (Eq. 3). In both cases, the results computed using the two procedures are in excellent agreement (cf. Figs. 2 and 3). Finally, in our evaluations of Eq. 3, for simplicity we chose to consider only the case in which the elements of $\boldsymbol{x}=\left[x_{1}, \ldots, x_{n}\right]$ are statistically independent. However, as we mentioned in the previous paragraph, the validity of Eq. 3 does not hinge on the independence of these elements.

A. The general formula (Eq. 3) specified for seven paradigms

Yes-no paradigm

In the yes-no paradigm, each trial presents a single stimulus, $\boldsymbol{x}=$ $x$, which belongs to either A or $\mathrm{B}$; the trial type A corresponds to a "yes" response, and B to a "no" response. We denote the conditional PDFs of the variable $x$ for the two trial types as 
$f_{x}(\boldsymbol{x} \mid A)$ and $f_{x}(\boldsymbol{x} \mid B)$. Assume that these trial types are equally likely a priori; plugging these expressions into Eq. 3 yields

$$
P c_{y e s-n o}=\frac{1}{2} \int_{x} \max \left\{f_{x}(x \mid A), f_{x}(x \mid B)\right\} d x
$$

For illustration, we convert the two PDFs to Gaussian PDFs, denoted as $f\left(x, \mu, \sigma^{2}\right)=\frac{1}{\sqrt{2 \pi \sigma^{2}}} e^{-\frac{(x-\mu)^{2}}{2 \sigma^{2}}}$, where $\mu$ is the mean, and $\sigma^{2}$ is the variance. Throughout our analysis, we set $\sigma^{2}=1$ and simplify the form of the Gaussian PDFs to $f(x-\mu)=\frac{1}{\sqrt{2 \pi}} e^{-\frac{(x-\mu)^{2}}{2}}$. We further set $\mu=\Delta$ for the trial type A and $\mu=0$ for the trial type B. Let $f_{x}(\boldsymbol{x} \mid A)=f(x-\Delta)$ and $f_{x}(\boldsymbol{x} \mid B)=f(x)$. Plugging them into Eq. 4, we obtain

$P c_{\text {yes }- \text { no }}^{\text {Gausian }}=\frac{1}{2} \int_{x=-\infty}^{\infty} \max \{f(x-\Delta), f(x)\} d x$.

\section{IFC paradigm}

In the 2IFC paradigm, each trial presents a pair of stimuli, $\boldsymbol{x}=\left[x_{1}, x_{2}\right]$, in the two observation intervals. The pair belongs to either the trial type AB ("signal in the 1 st interval") or BA ("signal in the 2 nd interval"). The conditional PDFs of the stimulus pair for the two trial types are $f_{x}(\boldsymbol{x} \mid A B)$ and $f_{x}(\boldsymbol{x} \mid B A)$. Assume that these trial types are equally likely a priori; Eq. 3 then becomes

$P c_{2 I F C}=\frac{1}{2} \iint_{x_{2}} \max \left\{f_{x}(x \mid A B), f_{x}(x \mid B A)\right\} d x_{1} d x_{2}$

To specify Eq. 6 for Gaussian distributions, we replace the two PDFs with Gaussian PDFs. The joint Gaussian conditional PDF is $f_{x}(\boldsymbol{x} \mid A B)=f\left(x_{1}-\Delta\right) f\left(x_{2}\right)$ for the trial type AB, and $f_{x}(\boldsymbol{x} \mid B A)=f\left(x_{1}\right) f\left(x_{2}-\Delta\right)$ for BA. Substituting them into Eq. 6, we obtain

$P c_{2 I F C}^{\text {Gaussian }}=\frac{1}{2} \int_{x_{2}=-\infty}^{\infty} \int_{x_{1}=-\infty}^{\infty} \max \left\{f\left(x_{1}-\Delta\right) f\left(x_{2}\right), f\left(x_{1}\right) f\left(x_{2}-\Delta\right)\right\} d x_{1} d x_{2}$.

\section{IFC paradigm}

In the 3IFC paradigm, each trial presents a trio of stimuli, $\boldsymbol{x}=$ $\left[x_{1}, x_{2}, x_{3}\right]$, in the three observation intervals. They belong to either the trial type ABB ("signal in the 1st interval"), BAB ("signal in the 2nd interval"), or BBA ("signal in the 3rd interval"). The conditional PDFs of the stimulus trio for the three trial types are $f_{x}(\boldsymbol{x} \mid A B B), f_{x}(\boldsymbol{x} \mid B A B)$, or $f_{x}(\boldsymbol{x} \mid B B A)$. Assume that these trial types are equally likely a priori; Eq. 3 becomes

$P c_{3 I F C}=\frac{1}{3} \iint_{x_{3}} \int_{x_{2} x_{1}} \max \left\{f_{x}(x \mid A B B), f_{x}(x \mid B A B), f_{x}(x \mid B B A)\right\} d x_{1} d x_{2} d x_{3}$.

Equation 8 can be specified for Gaussian distributions by replacing the three PDFs in the equation with the following three joint Gaussian PDFs:

$f_{x}(\boldsymbol{x} \mid A B B)=f\left(x_{1}-\Delta\right) f\left(x_{2}\right) f\left(x_{3}\right)$,

$f_{x}(\boldsymbol{x} \mid B A B)=f\left(x_{1}\right) f\left(x_{2}-\Delta\right) f\left(x_{3}\right)$,

$f_{x}(\boldsymbol{x} \mid B B A)=f\left(x_{1}\right) f\left(x_{2}\right) f\left(x_{3}-\Delta\right)$.

\section{Same-different paradigm}

In the same-different paradigm, each trial presents a pair of stimuli, $\boldsymbol{x}=\left[x_{1}, x_{2}\right]$, in the two observation intervals. The pair belongs to one of four possible trial types: AA, $\mathrm{BB}, \mathrm{AB}$, and $\mathrm{BA}$. The former two trial types, $\mathrm{AA}$ and $\mathrm{BB}$, correspond to the response "same," denoted $S$; the latter two, AB and BA, to the response "different," denoted $D$. We denote the conditional PDFs of the two-dimensional vectors of stimulus values, $\boldsymbol{x}=\left[x_{1}, x_{2}\right]$, for the four trial types as $f_{x}(\boldsymbol{x} \mid A A), f_{x}(\boldsymbol{x} \mid B B), f_{x}(\boldsymbol{x} \mid A B)$, and $f_{x}(\boldsymbol{x} \mid B A)$, respectively. Assuming that the four trial types are all equally likely a priori, the conditional composite PDF that $x$ comes from a "same" trial type is

$f_{x}(\boldsymbol{x} \mid S)=\left[f_{x}(\boldsymbol{x} \mid A A)+f_{x}(\boldsymbol{x} \mid B B)\right] / 2$.

The conditional composite PDF that $x$ comes from a "different" trial type is

$f_{x}(\boldsymbol{x} \mid D)=\left[f_{x}(\boldsymbol{x} \mid A B)+f_{x}(\boldsymbol{x} \mid B A)\right] / 2$.

Plugging these two PDFs into Eq. 3 yields

$P c_{\text {same-different }}=\frac{1}{2} \int_{x_{2}} \int_{x_{1}} \max \left\{f_{x}(x \mid S), f_{x}(x \mid D)\right\} d x_{1} d x_{2}$. 
To specify Eq. 14 for Gaussian distributions, we substitute $f_{x}(\boldsymbol{x}$ $\mid A A)=f\left(x_{1}-\Delta\right) f\left(x_{2}-\Delta\right)$ and $f_{x}(\boldsymbol{x} \mid B B)=f\left(x_{1}\right) f\left(x_{2}\right)$ into Eq. 12, $f_{x}(\boldsymbol{x} \mid A B)=f\left(x_{1}-\Delta\right) f\left(x_{2}\right)$ and $f_{x}(\boldsymbol{x} \mid B A)=f\left(x_{1}\right) f\left(x_{2}-\Delta\right)$ into
Eq. 13, and substitute both composite PDFs (Eqs. 12 and 13) into Eq. 14. The result is

$P c_{\text {same-different }}^{\text {Gaussian }}=\frac{1}{4} \int_{x_{2}=-\infty}^{\infty} \int_{x_{1}=-\infty}^{\infty} \max \left\{\left[f\left(x_{1}-\Delta\right) f\left(x_{2}-\Delta\right)+f\left(x_{1}\right) f\left(x_{2}\right)\right],\left[f\left(x_{1}-\Delta\right) f\left(x_{2}\right)+f\left(x_{1}\right) f\left(x_{2}-\Delta\right)\right]\right\} d x_{1} d x_{2}$

\section{The oddity paradigms}

In an $m$-interval $(m \geq 3)$, oddity paradigm, each trial presents $n=m$ stimuli, $\boldsymbol{x}=\left[x_{1}, \ldots, x_{m}\right]$, in the $m$ observation intervals. There are a total of $2 \times m$ possible trial types; for each trial type, one stimulus comes from one event, which can be either A or B, and the remaining $m-1$ stimuli come from the other event (either B or A). The task of the participant is to indicate which interval contains the "odd" stimulus. For the general $m$-interval oddity paradigm, the likelihood-ratio decision model can become quite complicated as $m$ exceeds four. Here, we consider only the simplest, threeand four-interval versions of the oddity paradigm, which are the most commonly used oddity paradigms in psychophysical studies. In the 3I case $(m=3)$, six trial types are possible, corresponding to three response alternatives, respectively. These are $\mathrm{ABB}$ and $\mathrm{BAA}$, corresponding to "The odd stimulus is in the first interval," denoted $O 1$; BAB and $\mathrm{ABA}$, corresponding to 02 ; and $\mathrm{BBA}$ and $\mathrm{AAB}$, corresponding to $O 3$. The conditional composite PDFs of $\boldsymbol{x}$ given these three response alternatives are:

$f_{x}(\boldsymbol{x} \mid O 1)=\left[f_{x}(\boldsymbol{x} \mid A B B)+f_{x}(\boldsymbol{x} \mid B A A)\right] / 2$,

$f_{x}(\boldsymbol{x} \mid O 2)=\left[f_{x}(\boldsymbol{x} \mid B A B)+f_{x}(\boldsymbol{x} \mid A B A)\right] / 2$,

$f_{x}(\boldsymbol{x} / O 3)=\left[f_{x}(\boldsymbol{x} \mid B B A)+f_{x}(\boldsymbol{x} \mid A A B)\right] / 2$.

Expressed in these conditional PDFs, Eq. 3 becomes

$P c_{3 \text { IIddity }}=\frac{1}{3} \iint_{x_{3}} \int_{x_{2}} \int_{x_{1}} \max \left\{f_{x}(x \mid O 1), f_{x}(x \mid O 2), f_{x}(x \mid O 3)\right\} d x_{1} d x_{2} d x_{3}$.

Similar derivations for the 4I oddity paradigm yield

$P c_{4 I \text { Iddity }}=\frac{1}{4} \int_{x_{4}} \cdot . \int_{x_{1}} \max \left\{f_{x}(x \mid O 1), f_{x}(x \mid O 2), f_{x}\left(x \mid O 3, f_{x}(x \mid O 4)\right\} d x_{1} \cdot \cdot d x_{4}\right.$.
For the 3I oddity paradigm, Eq. 19 can be specified for Gaussian distributions to obtain $P C_{3 \text { Ioddity }}^{\text {Gaussian }}$ by substituting into the equation the three conditional PDFs below:

$f_{x}(\boldsymbol{x} \mid O 1)=(1 / 2)\left[f\left(x_{1}-\Delta\right) f\left(x_{2}\right) f\left(x_{3}\right)+f\left(x_{1}\right) f\left(x_{2}-\Delta\right) f\left(x_{3}-\Delta\right)\right]$,

$f_{x}(\boldsymbol{x} \mid O 2)=(1 / 2)\left[f\left(x_{1}\right) f\left(x_{2}-\Delta\right) f\left(x_{3}\right)+f\left(x_{1}-\Delta\right) f\left(x_{2}\right) f\left(x_{3}-\Delta\right)\right]$,

$f_{x}(\boldsymbol{x} \mid O 3)=(1 / 2)\left[f\left(x_{1}\right) f\left(x_{2}\right) f\left(x_{3}-\Delta\right)+f\left(x_{1}-\Delta\right) f\left(x_{2}-\Delta\right) f\left(x_{3}\right)\right]$.

\section{Dual-pair paradigms}

As their name indicates, dual-pair paradigms involve the presentation of two pairs of stimuli, a total of four $(n=$ 4) stimuli, on each trial. Eight trial types are possible (AAAB, АABA, BBAB, BBBA, $\mathrm{ABAA}, \mathrm{BAAA}$, $\mathrm{ABBB}$, and $\mathrm{BABB}$ ), and two response alternatives that, as we explain below, depends on what version of the dual-pair paradigm is used. Here, we consider two versions that have been used in experiments, and for which the SDT decision model has been analyzed in previous publications (Creelman \& Macmillan, 1979; Micheyl \& Dai, 2008, 2009; Micheyl \& Messing, 2006; Noreen, 1981; Rousseau \& Ennis, 2001, 2002). In one version-namely the basic dual-pair paradigm, which is commonly abbreviated 4IAX (for four-interval, samedifferent) - the task of the participant is to indicate which pair contains stimuli that are different $(A B$ or $\mathrm{BA}$ vs. AA or BB). This version of the paradigm is useful in experiments that aim to measure performance in stimulus discrimination, or change detection (Micheyl et al., 2008; Semal \& Demany, 2006). In the second version-namely the 4IAX, AB-versus-BA paradigmthe exact same stimulus design can be used to explore a different ability of the participant, the ability to 
identify the direction of changes (i.e., $\mathrm{AB}$ vs. BA); all that the experimenter needs to do is to change the instructions. Specifically, the participant is now asked to indicate whether the pair containing one $\mathrm{A}$ and one $\mathrm{B}$ stimulus was $\mathrm{AB}$ or $\mathrm{BA}$. Although it is superficially similar to the basic 4IAX paradigm, the 4IAX, ABversus-BA paradigm involves a substantially different underlying decision strategy and a completely different relationship between $d^{\prime}$ and $P c$ (Micheyl \& Dai, 2009; Micheyl et al., 2008; Semal \& Demany, 2006).

For these two dual-pair paradigms, $P c$ can be determined following an approach similar to the one that we used for the two-interval same-different paradigm. The main differences are that the vector $\boldsymbol{x}$ now contains four elements, $\boldsymbol{x}=\left[x_{1}, x_{2}, x_{3}, x_{4}\right]$, instead of two, and that eight conditional PDFs (one for each of the four-interval stimulus sequences listed above), instead of four, need to be taken into account.

For the basic 4IAX paradigm, the conditional PDF of $\boldsymbol{x}$ given that the first pair was $\mathrm{AB}$ or $\mathrm{BA}$ - an event that we denote as $D 1$ to indicate that the pair containing the "different" stimuli was first-is $f_{x}(\boldsymbol{x} \mid D 1)=\left[f_{x}(\boldsymbol{x} \mid A B A A)+f_{x}(\boldsymbol{x} \mid A B B B)+\right.$ $\left.f_{x}(\boldsymbol{x} \mid B A A A)+f_{x}(\boldsymbol{x} \mid B A B B)\right] / 4$. Similarly, the conditional PDF of $\boldsymbol{x}$ given that the second pair was $\mathrm{AB}$ or $\mathrm{BA}$ - an event denoted as $D 2$-is $f_{x}(\boldsymbol{x} \mid D 2)=\left[f_{x}(\boldsymbol{x} \mid A A A B)+f_{x}(\boldsymbol{x} \mid A A B A)\right.$ $\left.+f_{x}(\boldsymbol{x} \mid B B A B)+f_{x}(\boldsymbol{x} \mid B B B A)\right] / 4$. After plugging these expressions into Eq. 3, we find

$P c_{4 I A X}=\frac{1}{2} \int_{x_{4}} . . \int_{x_{1}} \max \left\{f_{x}(x \mid D 1), f_{x}(x \mid D 2)\right\} d x_{1} . . d x_{4}$.

Equation 24 can be specified for Gaussian distributions to obtain $P C_{4 I A X}^{\text {Gaussian }}$ by substituting the following two conditional PDFs into the equation:

$$
\begin{aligned}
f_{x}(\boldsymbol{x} \mid D 1)= & (1 / 4)\left[f\left(x_{1}-\Delta\right) f\left(x_{2}\right) f\left(x_{3}-\Delta\right) f\left(x_{4}-\Delta\right)\right. \\
& +f\left(x_{1}-\Delta\right) f\left(x_{2}\right) f\left(x_{3}\right) f\left(x_{4}\right)+f\left(x_{1}\right) f\left(x_{2}-\Delta\right) f \\
& \left.\left(x_{3}-\Delta\right) f\left(x_{4}-\Delta\right)+f\left(x_{1}\right) f\left(x_{2}-\Delta\right) f\left(x_{3}\right) f\left(x_{4}\right)\right],
\end{aligned}
$$

$$
\begin{aligned}
f_{x}(\boldsymbol{x} \mid D 2)= & (1 / 4)\left[f\left(x_{1}-\Delta\right) f\left(x_{2}-\Delta\right) f\left(x_{3}-\Delta\right) f\left(x_{4}\right)\right. \\
& +f\left(x_{1}-\Delta\right) f\left(x_{2}-\Delta\right) f\left(x_{3}\right) f\left(x_{4}-\Delta\right)+f\left(x_{1}\right) f \\
& \left.\left(x_{2}\right) f\left(x_{3}-\Delta\right) f\left(x_{4}\right)+f\left(x_{1}\right) f\left(x_{2}\right) f\left(x_{3}\right) f\left(x_{4}-\Delta\right)\right] .
\end{aligned}
$$

For the 4IAX AB-versus-BA paradigm, the conditional PDF of $X$ given that one of the two presented pairs was $\mathrm{AB}$ - an event that we denote as DN for "downward," because as we noted above, $\mu_{\mathrm{A}}>\mu_{\mathrm{B}}$-is $f_{x}(\boldsymbol{x} \mid D N)=\left[f_{x}(\boldsymbol{x} \mid A B A A)+f_{x}(\boldsymbol{x} \mid A B B B)+f_{x}(\boldsymbol{x} \mid\right.$
$\left.A A A B)+f_{x}(\boldsymbol{x} \mid B B A B)\right] / 4$. Similarly, the conditional PDF of $X$ given that one of the two presented pairs was BA-an event denoted UP for "upward"-is $f_{x}(\boldsymbol{x}$ $U P)=\left[f_{x}(\boldsymbol{x} \mid B A A A)+f_{x}(\boldsymbol{x} \mid B A B B)+f_{x}(\boldsymbol{x} \mid A A B A)+\right.$ $\left.f_{x}(\boldsymbol{x} \mid B B B A)\right] / 4$. After plugging these expressions into Eq. 3, we find

$$
P c_{4 I A X_{A B} \text { vs } B A}=\frac{1}{2} \int_{x_{4}} . . \int_{x_{1}} \max \left\{f_{x}(x \mid D N), f_{x}(x \mid U P)\right\} d x_{1} . . d x_{4} .
$$

Likewise, Eq. 27 can be specified for Gaussian distribu-

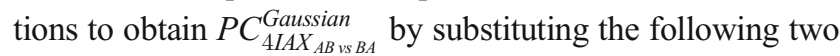
conditional PDFs into the equation:

$$
\begin{aligned}
f_{x}(\boldsymbol{x} \mid D N)= & (1 / 4)\left[f\left(x_{1}-\Delta\right) f\left(x_{2}\right) f\left(x_{3}-\Delta\right) f\left(x_{4}-\Delta\right)\right. \\
& +f\left(x_{1}-\Delta\right) f\left(x_{2}\right) f\left(x_{3}\right) f\left(x_{4}\right)+f\left(x_{1}-\Delta\right) f\left(x_{2}-\Delta\right) f \\
& \left.\left(x_{3}-\Delta\right) f\left(x_{4}\right)+f\left(x_{1}\right) f\left(x_{2}\right) f\left(x_{3}-\Delta\right) f\left(x_{4}\right)\right], \\
f_{x}(\boldsymbol{x} \mid U P)= & (1 / 4)\left[f\left(x_{1}\right) f\left(x_{2}-\Delta\right) f\left(x_{3}-\Delta\right) f\left(x_{4}-\Delta\right)\right. \\
& +f\left(x_{1}\right) f\left(x_{2}-\Delta\right) f\left(x_{3}\right) f\left(x_{4}\right)+f\left(x_{1}-\Delta\right) f\left(x_{2}-\Delta\right) f \\
& \left.\left(x_{3}\right) f\left(x_{4}-\Delta\right)+f\left(x_{1}\right) f\left(x_{2}\right) f\left(x_{3}\right) f\left(x_{4}-\Delta\right)\right]
\end{aligned}
$$

B. Evaluation of the general formula (Eq. 3) for 2IFC and 3IFC paradigms

The purpose of this section is twofold. First, we evaluate the accuracy of the proportion-correct $(P c)$ scores computed using the general formula implemented in MATLAB code (shown in the Appendix). Toward this aim, we have selected two special conditions for which true analytic solutions for computing the maximum $P c$ scores exist, so that the maximum $P c$ scores computed using the MATLAB code can be compared to exact results. Specifically, we computed $P c$ scores using the MATLAB code for the 2IFC paradigm using continuous Gaussian distributions, and for the 3IFC paradigm using discrete uniform distributions, and compared these scores to those obtained using the analytical solutions available in the SDT literature (see Green \& Swets, 1966, for computing $P c$ scores for 2IFC under Gaussian distributions, and Dai \& Kidd, 2009, for computing $P c$ scores for 3IFC under uniform distributions). Two types of applications for the MATLAB code are envisioned. In the first type, the researcher wants to compute the maximum $P c$ for a particular paradigm on the basis of a pair of basis probability functions that are known and continuous. For this type of application, these continuous 
basis PDFs will first need be converted to discrete PMFs before they are fed into the MATLAB code. Here we perform this conversion by digitizing the continuous PDFs into discrete PMFs. The conversion must be done properly to ensure that the discrete PMFs have sufficiently large numbers of bins (i.e., that the sampling resolution is sufficiently high) to closely approximate the original continuous PDFs and to produce accurate $P c$ scores. For this type of application, the computation of $P c$ scores with continuous Gaussian distributions (in the 2IFC section below) provides an example. In our evaluation, we will examine how the accuracy of the computed $P c$ scores depends on the number of bins allocated to the converted PMFs. The second type of application relates to situations in which the researcher wants to compute the maximum $P c$ for a particular paradigm on the basis of a pair of discrete probability mass functions ( $\mathrm{fA}$ and $\mathrm{fB}$ ) that we assume are already available to the researcher. These PMFs could be some known probability functions or be derived from some known PDFs (as we described above), or they could be determined empirically in some experiments. This type of application is straightforward; the researcher merely needs to specify the two basis functions as the inputs into the MATLAB code. The computation of $P c$ for discrete uniform distributions (in the 3IFC section below) provides an example of this type of application.

Second, we describe and illustrate how to use the MATLAB code of the general formula in steps. Briefly, the user of the MATLAB code provides the pair of basis PMFs of the stimuli as two column vectors, one named $\mathbf{f B}$, for the noise-alone or standard-alone event (B), and the other named fA, for the signal + noise or signal + standard event (A). Both vectors have the common size of $N b \times 1$, where $N b$ is the number of bins allocated to each PMF; both vectors are normalized so that each is summed to 1 . The user will save the two vectors in a mat file named $\triangle A B$. mat by executing the code save ('fAB.mat', ' $f A$ ', ' $f B$ '); in the MATLAB workspace. Next, the user will run the MATLAB code named max_pc.m; it returns the maximum $P c$ score in a variable named pcmax for the paradigm that the user has selected.

\section{IFC paradigm under continuous Gaussian distributions}

Following the steps outlined above, here we compute the maximum $P c$ scores for the 2IFC paradigm under continuous Gaussian distributions with equal variances. We begin by generating (by digitizing PDFs) the PMFs $\mathrm{fB}[\mathrm{N}(\mu \mathrm{B}=0, \sigma=1)]$ and $\mathrm{fA}[\mathrm{N}(\mu \mathrm{A}=\Delta, \sigma=1)$, where $\Delta$ is the difference in means, which also reflects the displacement between the two distributions. In all conditions, the range of $\mathrm{fB}$ is extended from $5 \sigma$ below to $5 \sigma+\Delta$ above its mean; the range of fA is extended from $5 \sigma+\Delta$ below to $5 \sigma$ above its mean. This evaluation using Gaussian distributions includes two parts. In the first part, we fixed the sampling resolution to 200 bins per $\sigma$ and compute the $P c$ scores for a range of values of $\Delta(0.125,0.5$, $1,1.5,2,2.5,3$, and 3.5 , in units of $\sigma$ ). For each condition, we obtained the result after saving the two PMFs $\mathrm{fB}$ and $\mathrm{fA}$ in fAB.mat, starting the MATLAB code max_pc.m, and selecting the 2IFC paradigm. The results obtained from the first part of the evaluation using continuous Gaussian distributions are plotted as circles in Fig. 2. For comparison, we also obtained the $P C$ values using the standard analytic solution of SDT for the 2IFC paradigm:

$P c_{2 \mathrm{IFC}}=\Phi(\Delta / \sqrt{2})$

where $\Phi$ is the cumulative Gaussian probability function and the displacement $\Delta$ is identical to the detectability index, $d^{\prime}$. The result is shown as the solid curve. It is worth noting that the equation above is equivalent to Eq. 7 for Gaussian distributions. Since the MATLAB code of the general formula here uses the discrete PMFs for $\mathrm{fA}$ and $\mathrm{fB}$, errors may arise from numerical integration. However, when the sampling resolution used for the PMFs is sufficiently high, the numerical errors are relatively small. For the present illustration, which uses 200 bins per $\sigma$ for the PMFs, the $P c$ scores computed using the MATLAB code of the general formula are

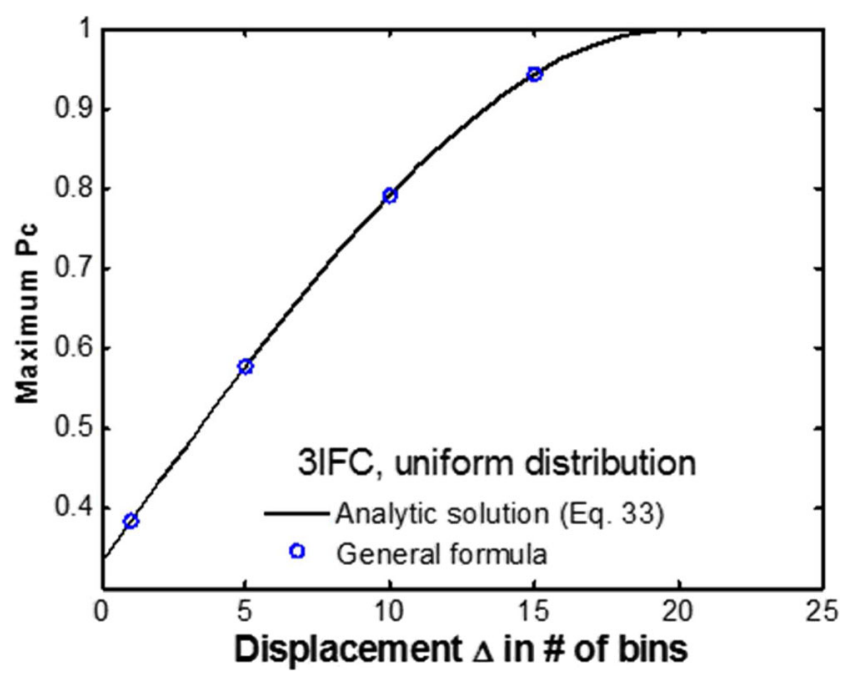

Fig. 3 Same as for Fig. 2, but for the 3IFC paradigm under discrete uniform distributions. The probability mass functions include 20 bins (for which the probability at each bin is $1 / 20$ ) and the number of bins (for which the probability at each bin is 0 ) corresponding to the displacement $(\Delta)$ between the probability distributions of the noisealone event (B) and the signal + noise event (A). In this plot, the $\Delta$ values on the $x$-axis are expressed as numbers of bins 
accurate to at least the sixth decimal place. The $P c$ scores obtained using the two procedures for common $\Delta$ values are virtually identical.

As an extra illustration of using the MATLAB code of the general formula, we examine how the maximum $P c$ obtained using probability distributions of a non-Gaussian family compares with that obtained from Gaussian distributions. For this illustration, we computed the $P c$ score for the 2IFC paradigm for Laplace distributions based on the following basis PDFs: $f_{x}(x \mid B)=\frac{1}{\sqrt{2}} e^{-\sqrt{2}|x|}$ and $f_{x}(x \mid A)=\frac{1}{\sqrt{2}} e^{-\sqrt{2}|x-1|}$, where both the means and the standard deviations are matched to Gaussian distributions in the condition of $\Delta=1$ above. Using a sampling resolution of 200 bins per $\sigma$, the computed maximum $P c$ is 0.793 for the Laplace distributions, as compared with the maximum $P c$ of 0.7603 for the Gaussian distributions.

In the second part of this evaluation using continuous Gaussian distributions, we fixed the displacement at $\Delta$ $=1$ and computed the $P c$ scores for several levels of sampling resolution $(10,20,40,80$, and 160 bins per $\sigma)$. The results indicated, not surprisingly, that the accuracy suffers as the sampling resolution is reduced from 160 to 10 bins per $\sigma$; the absolute error in the computed $P c$ scores increased by three orders of magnitude, from 0.0000001 to 0.0001 .

\section{IFC paradigm under discrete uniform distribution}

To evaluate the general formula for computing the maximum $P c$ scores for the 3IFC paradigm under the discrete uniform distribution, we defined the two probability mass functions (PMFs) as

$$
f B=\left\{\begin{array}{ll}
1 / n_{R}, & 1 \leq i \leq n_{R} \\
0 & \text { otherwise }
\end{array},\right.
$$

and

$$
f A=\left\{\begin{array}{lr}
1 / n_{R}, & \Delta+1 \leq i \leq n_{R}+\Delta \\
0, & \text { otherwise }
\end{array},\right.
$$

where $n_{R}$ is the number of bins of the uniform distribution and $\Delta$ is the displacement between the two PMFs measured in the number of bins. We evaluated the general formula for a fixed $n_{R}$ value ( $n_{R}=20$ bins), and a range of $\Delta$ values $(\Delta=1,5,10$, or 15 bins.) For each condition, we saved the two PMFs fB and $f A$ in $f A B$. mat, started the MATLAB code max pc.m, selected the 3IFC paradigm, and obtained the $P c$ score.

The results are plotted as the circles in Fig. 3. For comparison, we also computed the $P c$ values over values of $\Delta$ from 0 to 20 , using the formula of Dai \& Kidd (2009) for the 3IFC paradigm under the uniform distribution,

$P c_{3 I F C}^{\text {Uniform }}(\Delta)=\frac{1}{3}\left[1-\left(\frac{\Delta}{n_{R}}\right)^{3}\right]+\frac{\Delta}{n_{R}}$,

and plotted these values as the solid curve. The $P c$ scores computed using the two procedures are in excellent agreement.

Finally, the application of the general formula has a practical limitation, due to the limited memory capacity of the computing machine. For psychophysical tasks in which the number of stimuli presented on each trial is greater than two (i.e., $n$ $>2$, such as in the 3IFC, oddity, and dual-pair comparison paradigms), the size of the joint probability distribution of the stimuli can grow rapidly with $n$. For example, if the two probability mass functions $\mathrm{fB}$ and $\mathrm{fA}$ each have been allocated 2,000 bins, then the size of each joint probability distribution in a dual-pair comparison task, in which four stimuli are presented on each trial, will be as large as $2,000^{4}=1.6 \times 10^{13}$, which for many users, at least for the next few years, can drive the MATLAB program out of memory on their computers. Thus, the accuracy of the computed $P C$ scores, which is limited by the number of bins that can be allocated to representing each probability mass function, is ultimately limited by the memory capacity available on a particular computer.

\section{Summary and conclusion}

In this article, we have described and evaluated a general formula (Eq. 3) for computing the maximum $P c$ for various psychophysical paradigms for arbitrary probability distributions of sensory observations. Our evaluation of the formula in the context of two popular psychophysical paradigms - 2IFC and 3IFC - has showed that the computed $P c$ scores are highly accurate, even when the stimulus probability distributions are continuous, provided that care is taken to use a sufficiently large number of bins when converting the continuous PDFs into discrete PMFs. We hope that the MATLAB code implementing the general formula that is provided in the Appendix will facilitate applications of the approach described in this article.

Author note The authors are grateful to the Action Editor, J. A. Solomon, and to two anonymous reviewers for constructive comments and suggestions. 


\section{Appendix: MATLAB code for computing the maximum $P c$ for seven representative psychophysical paradigms with arbitrary probability distributions of the stimulus}

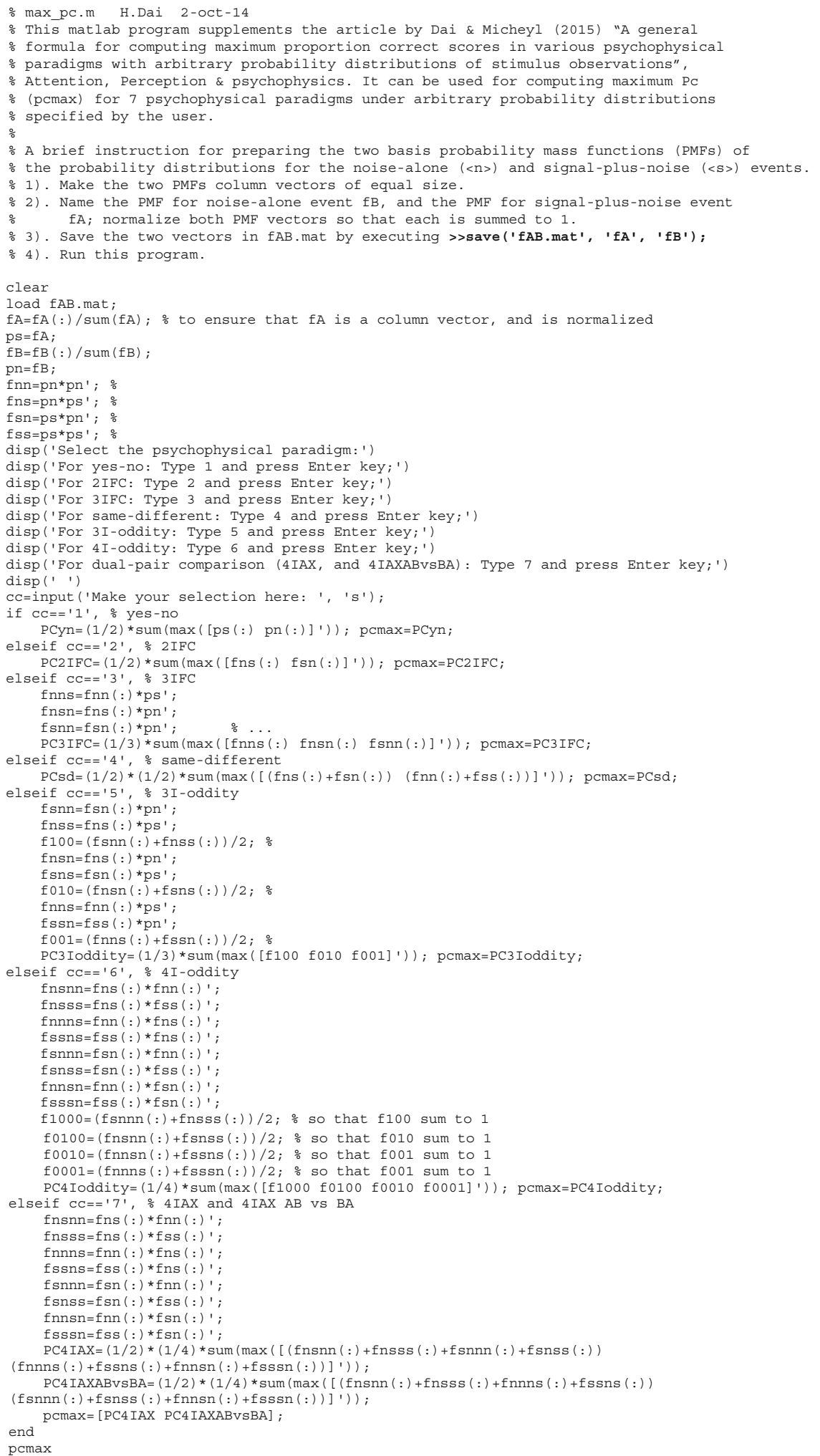




\section{References}

Berg, B. G., \& Green, D. M. (1990). Spectral weights in profile listening. Journal of the Acoustical Society of America, 88, 758-766.

Berliner, J. E., \& Durlach, N. I. (1973). Intensity perception: IV. Resolution in roving-level discrimination. Journal of the Acoustical Society of America, 53, 1270-1287.

Creelman, C. D. (1962). Human discrimination of auditory duration. Journal of the Acoustical Society of America, 34, 582-593.

Creelman, C. D., \& Macmillan, N. A. (1979). Auditory phase and frequency discrimination: A comparison of nine procedures. Journal of Experimental Psychology: Human Perception and Performance, 5, 146-156. doi:10.1037/0096-1523.5.1.146

Dai, H. (1994). Signal-frequency uncertainty in spectral-shape discrimination: Psychometric functions. Journal of the Acoustical Society of America, 96, 1388-1396.

Dai, H. (2008). On suppressing unwanted cues via randomization. Perception \& Psychophysics, 70, 1379-1382. doi:10.3758/PP.70.7. 1379

Dai, H., \& Green, D. M. (1993). Discrimination of spectral shape as a function of stimulus duration. Journal of the Acoustical Society of America, 93, 957-964.

Dai, H., \& Kidd, G. (2009). Limiting unwanted cues via random rove applied to the yes-no and multiple-alternative forced choice paradigms. Journal of the Acoustical Society of America, 126, 62-67.

Dai, H., \& Micheyl, C. (2010). On the choice of adequate randomization ranges for limiting the use of unwanted cues in same-different, dualpair, and oddity tasks. Attention, Perception, \& Psychophysics, 72, 538-547. doi:10.3758/APP.72.2.538

Dai, H., \& Micheyl, C. (2012). Separating the contributions of primary and unwanted cues in psychophysical studies. Psychological Review, 119, 770-788.

Dai, H., Nguyen, Q. T., \& Green, D. M. (1995). A two-filter model for frequency discrimination. Hearing Research, 85, 109-114.

Dai, H., Nguyen, Q. T., \& Green, D. M. (1996a). Decision rules of listeners in spectral-shape discrimination. Journal of the Acoustical Society of America, 99, 2298-2306.

Dai, H., Versfeld, N. J., \& Green, D. M. (1996b). The optimum decision rules in the same-different paradigm. Perception \& Psychophysics, $58,1-9$.

Danilova, M. V., \& Mollon, J. D. (2010). Parafoveal color discrimination: A chromaticity locus of enhanced discrimination. Journal of Vision, 10(1), 4.1-9. doi:10.1167/10.1.4

Dannemiller, J. L., \& Stephens, B. R. (1998). Contrast gain control in psychophysical contrast discrimination. Perception \& Psychophysics, 60, 1153-1163.

Geisler, W. S., Albrecht, D. G., Salvi, R. J., \& Saunders, S. S. (1991). Discrimination performance of single neurons: Rate and temporalpattern information. Journal of Neurophysiology, 66, 334-362.

Graham, N., Kramer, P., \& Yager, D. (1987). Signal-detection models for multidimensional stimuli: Probability distributions and combination rules. Journal of Mathematical Psychology, 31, 366-409.

Green, D. M. (1988). Profile analysis: Auditory intensity discrimination. New York, NY: Oxford University Press.

Green, D. M., \& Dai, H. (1991). Probability of being correct with 1 of $M$ orthogonal signals. Perception \& Psychophysics, 49, 100-101. doi: 10.3758/BF03211621

Green, D. M., \& Swets, J. A. (1966). Signal detection theory and psychophysics. New York, NY: Krieger.

Hall, J. W., \& Fernandes, M. A. (1983). The effect of random intensity fluctuation on monaural and binaural detection. Journal of the Acoustical Society of America, 74, 1200-1203.

Hecht, S., Shlaer, S., \& Pirenne, M. H. (1942). Energy, quanta, and vision. Journal of General Physiology, 25, 819-840.
Henning, G. B. (1966). Frequency discrimination of random amplitude tones. Journal of the Acoustical Society of America, 39, 336-339.

Irwin, R. J., \& Hautus, M. J. (1997). Likelihood-ratio decision strategy for independent observations in the same-different task: An approximation to the detection-theoretic model. Perception \& Psychophysics, $59,313-316$

Irwin, R. J., Hautus, M. J., \& Butcher, J. C. (1999). An area theorem for the same-different experiment. Perception \& Psychophysics, 61, 766-769.

Jesteadt, W., \& Bilger, R. C. (1974). Intensity and frequency discrimination in one- and two-interval paradigms. Journal of the Acoustical Society of America, 55, 1266-1276.

Kidd, G., Jr., Mason, C. R., Brantley, M. A., \& Owen, G. A. (1989). Roving-level tone-in-noise detection. Journal of the Acoustical Society of America, 86, 1310-1317.

Kidd, G., Jr., Mason, C. R., Uchanski, R. M., Brantley, M. A., \& Shah, P. (1991). Evaluation of simple models of auditory profile analysis using random reference spectra. Journal of the Acoustical Society of America, 90, 1340-1354.

Lutfi, R. A., \& Doherty, K. A. (1994). Effect of component-relativeentropy on the discrimination of simultaneous tone complexes. Journal of the Acoustical Society of America, 96, 3443-3450. doi: $10.1121 / 1.409022$

Lutfi, R. A., Doherty, K. A., \& Oh, E. (1996). Psychometric functions for the discrimination of spectral variance. Journal of the Acoustical Society of America, 100, 2258-2265.

Macmillan, N. A., \& Creelman, C. D. (2005). Detection theory: A user's guide (2nd ed.). Mahwah, NJ: Erlbaum.

May, K. A., \& Solomon, J. A. (2013). Four theorems on the psychometric function. PLoS ONE, 8, e74815. doi:10.1371/journal.pone.0074815

Micheyl, C., \& Dai, H. (2008). A general area theorem for the samedifferent paradigm. Perception \& Psychophysics, 70, 761-764. doi: 10.3758/PP.70.5.761

Micheyl, C., \& Dai, H. (2009). Likelihood ratio, optimal decision rules, and relationship between proportion correct and $d^{\prime}$ in the dual-pair AB-versus-BA identification paradigm. Attention, Perception, \& Psychophysics, 71, 1426-1433. doi:10.3758/APP.71.6.1426

Micheyl, C., Divis, K., Wrobleski, D. M., \& Oxenham, A. J. (2010). Does fundamental-frequency discrimination measure virtual pitch discrimination? Journal of the Acoustical Society of America, 128, 1930-1942.

Micheyl, C., Kaernbach, C., \& Demany, L. (2008). An evaluation of psychophysical models of auditory change perception. Psychological Review, 115, 1069-1083.

Micheyl, C., \& Messing, D. P. (2006). Likelihood ratio, optimal decision rules, and correct response probabilities in a signal detection theoretic, equal-variance Gaussian model of the observer in the 4IAX paradigm. Perception \& Psychophysics, 68, 725-735. doi:10.3758/ BF03193696

Moore, B. C. J., \& Glasberg, B. R. (1989). Mechanisms underlying the frequency discrimination of pulsed tones and the detection of frequency modulation. Journal of the Acoustical Society of America, $86,1722-1732$.

Morgan, M. J. (2005). The visual computation of 2-D area by human observers. Vision Research, 45, 2564-2570.

Nachmias, J. (1999). How is a grating detected on a narrowband noise masker? Vision Research, 39, 1133-1142.

Nachmias, J. (2002). Contrast discrimination with and without spatial uncertainty. Vision Research, 42, 41-48.

Nachmias, J., \& Kocher, E. C. (1970). Visual detection and discrimination of luminance increments. Journal of the Optical Society of America, 60, 382-389.

Neri, P. (2013). The statistical distribution of noisy transmission in human sensors. Journal of Neural Engineering, 10, 016014.

Noreen, D. L. (1981). Optimal decision rules for some common psychophysical paradigms. In S. Grossberg (Ed.), Mathematical 
psychology and psychophysiology (Proceedings of the Symposium in Applied Mathematics of the American Mathematical Society and the Society for Industrial and Applied Mathematics, Vol. 13, pp. 237-279). Providence, RI: American Mathematical Society.

Oxenham, A. J., \& Buus, S. (2000). Level discrimination of sinusoids as a function of duration and level for fixed-level, roving-level, and across-frequency conditions. Journal of the Acoustical Society of America, 107, 1605-1614.

Regan, D., \& Hamstra, S. (1991). Shape discrimination for motiondefined and contrast-defined form: Squareness is special. Perception, 20, 315-336.

Regan, D., \& Hamstra, S. (1992). Shape discrimination and the judgment of perfect symmetry: Dissociation of shape from size. Vision Research, 32, 1845-1864.

Rieke, F., Warland, D., de Ruyter van Steveninck, R., \& Bialek, W. (1999). Spikes: Exploring the neural code. Cambridge, MA: MIT Press.

Rousseau, B., \& Ennis, D. M. (2001). A Thurstonian model for the dual pair (4IAX) discrimination method. Perception \& Psychophysics, 63, 1083-1090.
Rousseau, B., \& Ennis, D. M. (2002). The multiple dual-pair method. Perception \& Psychophysics, 64, 1008-1014.

Semal, C., \& Demany, L. (2006). Individual differences in the sensitivity to pitch direction. Journal of the Acoustical Society of America, 120, 3907-3915.

Spiegel, M. F., Picardi, M. C., \& Green, D. M. (1981). Signal and masker uncertainty in intensity discrimination. Journal of the Acoustical Society of America, 70, 1015-1019.

Strickland, E. A., \& Dhar, S. (2000). An analysis of quasi-frequencymodulated noise and random-sideband noise as comparisons for amplitude-modulated noise. Journal of the Acoustical Society of America, 108, 735-742.

Versfeld, N. J., Dai, H., \& Green, D. M. (1996). The optimum decision rules for the oddity task. Perception \& Psychophysics, 58, $10-21$.

Webster, M. A., De Valois, K. K., \& Switkes, E. (1990). Orientation and spatial-frequency discrimination for luminance and chromatic gratings. Journal of the Optical Society of America A, 7, 1034-1049. 